\title{
Dual-Band Sierpinski Fractal Monopole Antenna
}

\author{
J. Soler", J. Romeu
}

Department of Signal Theory and Communications Universitat Politècnica de Catalunya (UPC) c/ Jordi Girona 1-3, Campus Nord Edifici D-3, 08034 Barcelona, Spain - Email: jsoler@tsc.upc.es

\begin{abstract}
A new top-loaded reduced-sized dual-band monopole antenna for wireless communications is presented. The antenna provides better than $-15 \mathrm{~dB}$ input return loss and keeps the same radiation pattern over both bands. In addition, a low-profile performance is achieved.
\end{abstract}

\section{Introduction}

There is a considerable interest in developing dual-band antennas since they permit telecom operators to reduce their costs and to minimise the environmental impact. Up until the present time, various designs have been proposed [1-2]. Contrary to the present prototype, the mentioned designs do not only use one radiating element, which operates simultaneously at two different bands. A top-loaded monopole is considered to obtain a dual-band and a low-profile performance. In particular, a top-loading triangular element is added to a modified Sierpinski gasket antenna [3].

There is a growing interest in DECT technology as it matches the needs of both residential and business users in urban, suburban and sparsely populated areas. Consequently, several new bands have been recently assigned to this technology. In particular, to the existent $1.88-1.9 \mathrm{GHz}$ band, three new bands have been added; that are, 1.9-1.92, 1.91-1.93 and 3.4-3.6 GHz. In the present study, a dual-band DECT $(1.9 \mathrm{GHz})$-DECT $(3.5 \mathrm{GHz})$ reduced-size antenna which provides coverage of all the DECT bands is studied. In this paper, the most relevant results for the dual-mode monopole antenna are presented.

\section{Antenna configuration}

The antenna, depicted in Fig. 1, consists of a one-iteration modified Sierpinski gasket with a triangular top-loading loop element. The antenna is printed over a thin dielectric substrate and fed using a microstrip matching network, which is fed by means of a coaxial probe, Fig 2 . The matching network consists of two series transmission lines. This top-loaded novel configuration allowed the antenna to have a more reduced size than the obtained when the design was performed using a Sierpinski gasket monopole [4]. In particular, a $40-45 \%$ in size reduction can be obtained.

\section{Experimental results}

The input reflection coefficient of the antenna relative to $50 \Omega$ was measured using an HP8510B. The measured input Voltage Standard Wave Ratio (VSWR) is plotted in Fig. 3. The antenna provides better than 1.43:1 input VSWR; that is, $-15 \mathrm{~dB}$ return loss, over both DECT bands (1.88-1.93 GHz and 3.4-3.6 GHz). Thus, an optimum 
impedance match is obtained for both bands. The bandwith at the lower band is $2.6 \%$ and at the higher band is $5.7 \%$.

The radiation patterns of the antenna were measured in an anechoic chamber. The maximum directivity of the monopole was calculated using the measured data, being 5 $\mathrm{dB}$ at $1.905 \mathrm{GHz}$ and $6.8 \mathrm{~dB}$ at $3.5 \mathrm{GHz}$.

In Fig. 4 the horizontal and vertical cuts of the radiation patterns at the center frequencies are shown: at $1.905 \mathrm{GHz}$ in the first row, and at $3.5 \mathrm{GHz}$ in the second The antenna is vertically $\left(\mathrm{E}_{\theta}\right)$ polarised and presents low levels of cross-polarization. At the first band, the horizontal plane is omnidireccional and the vertical plane corresponds to a typical dipole radiation pattern with a finite ground plane. At the second band, the radiation pattern is similar to that of the first band. A slight difference is observed in the vertical plane at $3.5 \mathrm{GHz}$ in the broadside direction. This pattern variance could be caused by the spurious radiation of the matching network.

\section{Conclusion}

A novel dual-band top-loaded reduced-size monopole antenna has been presented. With the triangular top-loading loop element the spacing between bands can be adjusted. Furthermore, a 40-45\% size-reduction factor, compared with the Sierpinski gasket monopole, can be obtained. Due to the growing interest in DECT technology, a prototype for this system was developed. The same radiation pattern is kept among the two bands and a good impedance match is also obtained. Advances in this antenna are currently been developed. It has been demonstrated that this novel top-loaded configuration constitutes an alternative to design dual-band monopole antennas.

\section{Acknowledgements}

This work has been financially supported by CICYT and the European Commission through grant FEDER 2FD97-0135.

\section{References}

[1] D.Liu, "A Dual-band antenna for cellular applications", IEEE Antennas and Propagation Society International Symposium and URSI Radio Science Meeting Proceedings, vol. 2, pp786-789, June 21-26, 1998, Atlanta.

[2] P.Haapala, P. Vainikainen, P. Eratuuli, "Dual frequency helical antennas for handsets", IEEE VTS 46th Vehicular Technology Conference Proceedings vol. 1 00.336-338, April 28-May 1, 1996, Atlanta, GA.

[3] C.Puente, J.Romeu, R.Bartolome, R.Pous, "Perturbation of the Sierpinski antenna to allocate operating bands", IEE Electronics Letters, vol. 32, no. 24, pp. 2186-2187, November 1996.

[4] C.Puente, J.Romeu, R.Pous, X.Garcia, F.Benitez, "Fractal multiband antenna based on the Sierpinski gasket", IEE Electronics Letters, January 1996, Vol. 32, no. 1, pp. 12. 


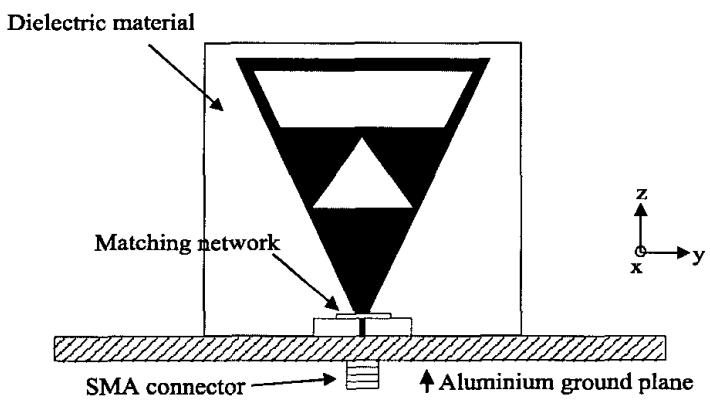

Figure 1: Dual-band monopole configuration. Front view

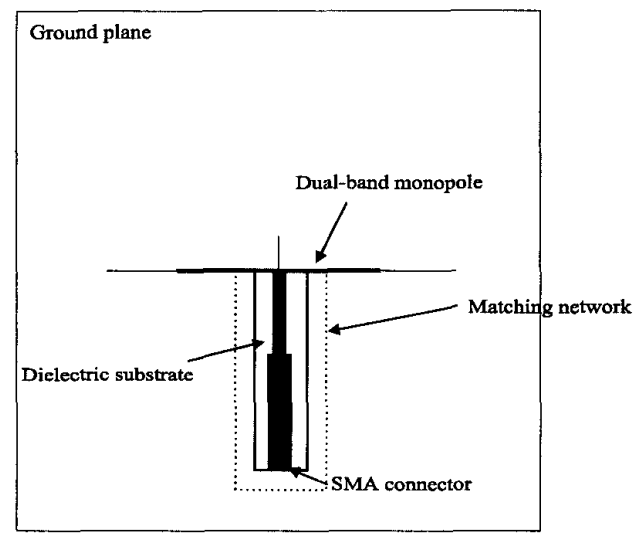

Figure 2: Dual-band monopole configuration. Up view 

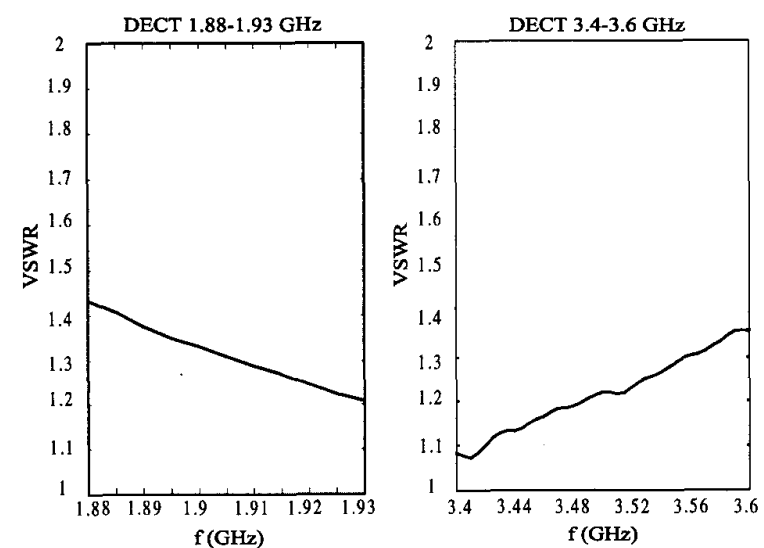

Figure 3: Measured input VSWR

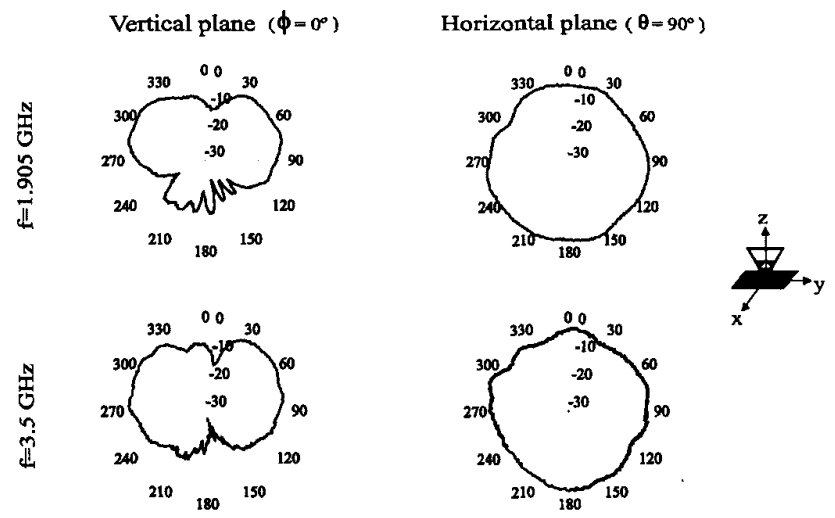

Figure 4: Horizontal and vertical planes of radiation patterns 